\title{
The Social Media Intelligence Analyst for Emergency Management
}

\author{
Robert Power \\ Data61 CSIRO \\ robert.power@data61.csiro.au
}

\author{
Justin Kibell \\ Emergency Management Victoria \\ justin.kibell@emv.vic.gov.au
}

\begin{abstract}
The Social Media Intelligence Analyst is a new operational role within a State Control Centre in Victoria, Australia dedicated to obtaining situational awareness from social media to support decision making for emergency management. We outline where this role fits within the structure of a command and control organization, describe the requirements for such a position and detail the operational activities expected during an emergency event. As evidence of the importance of this role, we provide three real world examples where important information was obtained from social media which led to improved outcomes for the community concerned.

This is the first time a dedicated role has been formally established solely for monitoring social media for emergency management intelligence gathering purposes in Victoria. To the best of our knowledge, it is also the first time such a dedicated position in an operational crisis coordination centre setting has been described in the literature.
\end{abstract}

\section{Introduction}

The term 'social media' refers to internet-based applications that enable people to communicate and share resources and information. Widely used and popular examples include blogs, discussion forums, chat rooms, wikis, Instagram, YouTube, LinkedIn, Facebook and Twitter. In an emergency everyone is a possible source of valuable information to both gather and distribute content [1,2,3]. Information flows rapidly on social media and this can be used to the advantage of emergency managers so long as they have the tools and knowledge to capitalize on this relatively new but pervasive medium $[1,4,5,6]$.

The continuous and connected nature of social media means online-networks and their users may have more information about an unfolding disaster than the dedicated response teams $[3,7,8]$. Harnessing this shared information, notably the images and videos posted by the community [9], provides unparalleled insights into events as they unfold. With this new channel of information emergency management agencies are able to detect new issues, engage communities and react accordingly $[10,11]$.

Proactively monitoring and engaging with the social media community also helps to identify trends, issues and urgent needs with regard to official messaging, misinformation and questions that require a response $[1,2,3]$.

Social media is now part of the fabric of society, exemplified by how many people communicate and share information. Emergency management agencies need to embrace, enhance and embed the use of social media before, during and after emergencies, and to challenge the view that social media is only about information output, such as publishing warnings and information updates, as it is rich with information they should know [12,13,14]. One of the barriers to effectively using social media content for emergency management purposes is the lack of formal policies and procedures regarding its use and a sceptical view and lack of trust for anything sourced from social media $[2,14,15]$.

This paper outlines the roles and responsibilities of the Social Media Intelligence Analyst at the State Control Centre (SCC) operated by Emergency Management Victoria (EMV) in Australia. By sharing our experience it is hoped other agencies will consider the value of adopting similar arrangements. We also define the requirements, social media information needs and daily work practices supporting this role which will be of use to researchers and tool developers.

\section{Background}

\subsection{Emergency Management Victoria}

Emergency Management Victoria (EMV) is a statutory body established in July 2014 by the State Government of Victoria to ensure the state is prepared for and can respond to emergencies and is able to recover more quickly from their impacts. Victoria's emergency services organisations, such as the police force, fire brigade, state emergency service and ambulance service, are managed as separate entities. 
Victoria has historically suffered from extreme fire events causing loss of life. In 2009, 173 people died, 78 towns were impacted and losses estimated at A\$2.9 billion resulted from a number of fires, collectively referred to as the Black Saturday bushfires. There were also around 5000 injured and over 2000 homes destroyed [16]. This was the worst fire disaster in Australia's history. Many challenges were identified from this event [17] and the EMV was established partly as a result in order to better manage such events in the future.

\subsection{State Control Centre}

The State Control Centre (SCC), located in Melbourne under the management of EMV, is the primary control centre for the management of emergencies in Victoria. The SCC is the central point for a network of regional and incident control centres across the state. The SCC is tasked with:

- maintaining situational awareness to support strategic decision-making

- sharing information and engaging with key stakeholders

- ensuring readiness arrangements are in place

- ensuring control strategies and arrangements are appropriate

- $\quad$ ensuring incident progression is predicted

- $\quad$ ensuring information and community warnings are timely and appropriate

- $\quad$ prioritising the allocation of state and specialist resources

- $\quad$ providing support to State, regional, incident control and agency personnel

\subsection{Motivation}

Social media has a role to play in all phases of the emergency management life cycle; before, during and after emergency events $[1,7,12]$. The management of emergency situations requires time critical decision making, usually based on limited knowledge which is incrementally revealed as the situation progresses and more information filters through to the decision makers $[18,19]$. In these circumstances, outcomes are highly sensitive to imprecise knowledge and it is in this context that social media serves as an additional realtime information source to support decision making during the fast-paced demands of disaster response. Additionally, social media content can be combined with other, more traditional information sources, to enhance situational awareness, providing further context to traditional information channels, and serves as a means to corroborate, act upon or contradict incorrect information [2].

While analysis of information on social media can provide insight into the community experience of a situation, for example through trending keywords and topics, the inherent characteristics of this big data source, notably the three V's of volume, variety and velocity, make such analysis problematic for the time critical nature of emergency management where the imperative is to act quickly. This situation is exacerbated by the preference for information to be detailed, accurate and verified.

For these reasons, social media content needs to be managed in ways different to sources of information traditionally used for emergency management purposes. These considerations are succinctly summarized as [1]:

- the degree of detail and verification needed

- methods of aggregation and filtering

- challenges associated with volume and information access

- $\quad$ appropriate search parameters

- data management considerations such as data format, update frequency, storage, and removal of personally identifiable information

- restrictions and challenges associated with the adoption of third-party technologies with respect to legacy technology platforms

- $\quad$ security and privacy implications

These issues will vary depending on the characteristics of the event, for example the severity, type of incident, its location, the proximity to communities and duration. Similarly, the urgency to communicate information will again depend on these issues, as well as the needs of the information recipients, such as first responders, search and rescue, relief coordination, recovery efforts, informing impacted communities and so on.

\subsection{Method}

This work is a result of two face to face interviews with the subject matter expert using open ended questions focused on understanding the role and activities of the Social Media Intelligence Analyst at the SCC. Notes were taken during the interviews, the first also being recorded, with follow up questions fielded through email and phone calls. These interviews were part of a wider investigation into the use of social media by three emergency management organisations in Australia [3]. During this process, the EMV was identified as being an advanced adopter of intelligence gathering from social media. Much of the material reported here aligns with the EMV social 
media training material and draws upon direct operational experience of the social media intelligence analyst role.

\section{Key Drivers}

The key drivers for information gathering from social media platforms are similar to that of other information channels monitored by SCC Intelligence Analysts which are described below.

\subsection{Situational Awareness}

Situational Awareness is understanding what's happening at a given place and time, usually 'now', and using this information to predict likely future outcomes. In order to improve situational awareness, relevant, current and accurate information is needed. Social media can help in this context by accessing eyewitness accounts of what's happening, essentially providing eyes and ears on the ground from the community who happen to be nearby and reporting what they are experiencing as the emergency unfolds.

Photos and videos provide an unbiased account of the situation. Text messages may be subjective, based on the author's viewpoint and personal experiences. A photo is not encumbered in this way and is a valuable source of situational awareness. Similarly, videos are a high value source of information. High profile individuals, notably journalists, are a more reputable source of information and can be targeted to obtain more reliable content than that of the general public.

\subsection{Hazard/Threat Behaviour}

Social media can help identify emerging risks or possible consequences of imminent events. For certain events, such as severe storms, existing known networks can be relied upon for information, such as storm chasers, spotters and enthusiasts. As noted previously, photos and videos are especially useful for such events. For example, photos of bushfires that show the smoke direction, colour, location and point of origin can be used to provide configuration parameters to fire modelling software to help predict fire behaviour. Similarly, photos of flood events can be used to verify predictions using on-scene observations. Community provided information can also be used to corroborate other observations such as those from sensor readings.

\subsection{Impacts}

The main purpose of emergency management is to respond to situations, ensuring safety of citizens, restoring impacted services and making the situation safe. A key component of these activities is to understand the impact to the community due to the emergency event. Reports on social media can help to understand how people in the vicinity are reacting and can also be used to identify additional risks or potential consequences that may arise. For example, what are their actions: are people self-evacuating or staying in place? What are the influential social media accounts, such as those belonging to news media, journalists and talk back radio hosts, reporting?

Key issues to be monitored include infrastructure failure (power outages, exposed sewerage, burst water mains) transportation issues (traffic accidents, signal failures, light rail or train derailments) built environment incidents (building or bridge collapse) and issues arising from the emergency event itself (smoke coverage, flood waters, storm damage).

\subsection{Weather}

Weather predictions are closely monitored during extreme weather events such as severe storms and cyclones. These predictions can be corroborated from community advice such as reports of hailstones, wind speed and rain intensity. When these reports are geotagged, the storm front can be monitored in near real-time. For cyclones, people report the calm experienced as the eye of the storm passes over them: a short respite from the extreme wind and rain that quickly resumes as the cyclone moves on.

As noted before, such observations can be used to assist analysts to calibrate their modelling to improve predictions. For example, social media photos showing hailstone sizes at certain locations can assist with forecast accuracy and value add to warnings being broadcast noting hailstone sizes at reported locations.

\subsection{Early Advice}

Some emergency events, such as floods and cyclones, occur with advanced warning based on weather conditions allowing accurate predictions and warnings to be issued. Similarly, the conditions leading up to the summer months are carefully monitored, such as the fuel load in the environment and soil moisture content, as well as long term weather trends, to predict the likely event of bushfires.

There are however many events that occur with no warning at all, such as earthquakes, flash floods, fires and also events that are a result of human induced hazards such as terrorist incidents, criminal activity and traffic accidents. For these events, early observations may be reported on social media that 
provide initial advice to the emergency services. These reports may include additional information that could be critical for informed decision making in the early stages of incident control activities.

\subsection{Sentiment}

The reports made on social media should be assessed in terms of the sentiment or emotion behind the message. As well as providing information about the event, the impact to the community, the event conditions and so on, how are those affected reacting to the emergency emotionally or psychologically?

Another important aspect is to determine how the community feels about how the emergency services are responding. Reputational damage can occur unintentionally when comments are made without full knowledge of how the response is being coordinated or it could be useful insight into a breakdown of communication within the response agencies. In either case, it is important to identify, investigate and follow up such reports and inform the community from an official source that the situation is being managed and that appropriate actions are being undertaken.

\subsection{External Context}

While the primary drivers for intelligence gathering from social media have been outlined above, a final aspect is to monitor what is going on in neighbouring jurisdictions. This can be useful to prepare for the possibility of providing assistance to help fellow response agencies deal with large scale disaster events.

\section{The Social Media Intelligence Analyst}

The role of the SCC Social Media Intelligence Analyst working within the SCC Intelligence Section is to provide the intelligence function with a dedicated social media intelligence capability. This specialist role monitors, collects, processes, analyses and disseminates key information found on social media and open source networks to address the priority intelligence requirements.

\subsection{Organisation}

The Social Media Intelligence Analyst is not an officially recognised role within the emergency management standards in use within Australia: the Australian Emergency Management Manual series [18] and Australasian Inter-Service Incident Management System (AIIMS-4) [19]. Nor is it a recognized specific role within current Victorian emergency management doctrine. This capability does however fall within the role of the Intelligence Officer or a member of the Situation and Analysis Unit in the AIIMS-4 structure.

The SCC is organised in a hierarchical structure from the Emergency Management Commissioner at the top, through to sections with general areas of concern, such as planning, logistics, investigation, public information and so on. These sections are divided into units that have a specific focus.

The position of Social Media Intelligence Analyst was established in October 2014 and is still under ongoing development. This position falls into the Intelligence Analyst Unit within the Intelligence Section. This section is managed by the SCC Intelligence Officer whose role is to coordinate the different aspects of operational intelligence gathering, of which social media forms one part. Within the Intelligence Section there are also units dedicated to GIS mapping, fire and flood modelling, meteorologists and liaison officers to manage interactions with other agencies, such as the police, education, utilities, public transport and similar government organisations.

To facilitate information sharing within the Intelligence Section, people within these units work closely together. They are physically co-located in the control room and each role has a uniquely coloured brassard as a means of identification. For example, the SCC Social Media Intelligence Analyst wears an orange coloured brassard with 'Social Media' written on it so they can be readily recognised when needed.

\subsection{Requirements}

The Social Media Intelligence Analyst role is mainly focused around the early identification of threats or emergencies reported on social media and for gaining enhanced situational awareness about known incidents underway. Once relevant information is identified, it needs to be verified. This can be achieved by checking the authenticity of the source, looking for accompanying images or videos to corroborate the report, and by checking if the information is also being reported by other users, in close proximity if possible, or on different social media platforms.

A corollary to the verification task is one of myth busting: correcting false reports and rumours. Such information can be spread on social media inadvertently by people who are misinformed or in some cases is a result of malicious activity. In either case, once identified, this misinformation needs to be corrected. Being a trusted source of information to the community about emergency events is one of the main roles of the SCC: in times of disasters the emergency services are relied upon to provide accurate and timely information to the community. 
Note that this is not the role of the Social Media Intelligence Analyst - at the SCC it is a function of the Social Media Officer within the Media Unit of the Public Information Section. The activities of this role include providing approved and verified information through official social media accounts, monitoring these social media accounts for questions and responding accordingly, scanning the social media landscape to determine information is being distributed correctly and effectively online and correcting misinformation.

Obviously the Social Media Intelligence Analyst and the Social Media Officer work closely together.

Another important task is to understand how the incident is being described or reported on social media: what are the relevant hashtags, keywords, handles, and websites being used? This information can be used to refine search terms to identify relevant information and to prime the investigation on other platforms. Knowing the correct query terms to find content is an important task in social media mining in this context.

The information found on social media about an incident can be analysed to gauge the community reaction. Such sentiment or emotional analysis can give insight to how the community will respond to the event as it unfolds and possibly escalates in severity. For example, are people taking the threat seriously; are they heeding warnings provided; are they preparing for the event, for example by evacuating or enacting their own emergency management plans? There can also be important community reactions about the activities of the response agencies, for example, are they meeting expectations? This information can be useful to improve the community messaging about the event. This analysis can be difficult since the aim is to establish the sentiment of the people affected by the emergency event, not those simply commenting on it.

Another recognised area of importance is to identify who the key influencers within the social media networks are. Specifically monitoring these accounts, and possibly their followers, can be beneficial since they have impact within their networks. This can be done by establishing who these individuals or entities are within specific regions. For example, local journalists, politicians or celebrities can often have a dedicated following on social media. Similarly, local media outlets (newspapers, radio and TV stations), community groups and not for profit organisations have an online presence and follower network. Monitoring these accounts can give greater insight into what the potentially impacted community are experiencing.

The EMV have established a list of such influential accounts by region across the state. These key accounts are followed during times of emergency and the list is actively maintained. Dynamically identifying who these key influencers are during an emergency event is more difficult and to our knowledge has not been effectively done during an incident response. This is an area of future research.

\subsection{Information Dissemination}

One of the principles of emergency management is to deliver the right information in the right format to the right people at the right time. Once information from social media has been found and verified, it needs to be provided to the appropriate stakeholders in a timely fashion. This is coordinated by the SCC Intelligence Officer: it is their judgement to evaluate the social media information found in context with other information in order to develop a common operating picture of the unfolding situation and provide this information to the appropriate people. This could be in the form of situation reports, briefings, a phone call or simply an entry into a common online log accessible to those throughout the SCC and beyond.

\subsection{Summary}

In summary, the Social Media Intelligence Analyst is focused on:

- early identification of threats or emergencies within social media information networks

- gaining enhanced situational awareness using information found within social media and open source information networks

- validating information found

- sourcing images and video content

- using validated social media information to assist in corroborating information from other information sources

- coordinating information gathering via crowd sourcing techniques when required

- determining what are the relevant handles, hashtags and websites being used for current events of interest

- working closely with the Social Media Officers

- analysing community sentiment and reaction

- myth busting

- establishing who are the key influencers and what are they saying

- ensuring social media intelligence is disseminated to the appropriate stakeholders in a timely fashion

- contributing social media information and subsequent intelligence to the products and briefings prepared by the Intelligence Section 
- maintaining the sources and agencies database of influencers, hashtags, websites as a common knowledge base

\section{Operational Tasks}

There are many elements to the Social Media Intelligence Analyst role. To assist with understanding what a typical day might involve a daily running sheet has been developed to outline specific tasks with associated times in a checklist format. These daily activities are divided into three phases: arrival, monitoring and collaboration, and handover.

\subsection{Arrival}

Upon arrival, the Social Media Intelligence Analyst prepares for a shift by first orientating themselves to the current situations being monitored. This is done through a hand over with the previous analyst, if available, in reference to the log book and by checking in with the SCC Intelligence Officer. This briefing identifies what the significant incidents or issues are, the key hazards, the priority social media information required and the key outputs and meetings needed in the short term. A briefing is also undertaken with the Social Media Officer in the Public Information team to identify the current trends occurring on the social media channels managed by the SCC.

Once this orientation has been done, the intelligence monitoring systems are activated and configured appropriately to meet the demands of the anticipated tasks identified as part of the arrival orientation. This configuration is performed by planning the monitoring activities for the shift: what keywords and hashtags should be used for searches and who are the key influencers that should be monitored?

The SCC makes use of a large suite of tools to support their monitoring activities. These range from freely available services (Facebook, Instagram, Twitter, TweetDeck, SocialMention, FotoForensics, TinEye, Storify, Pipl, YouTube and more), commercially available social media monitoring tools, free open source software (EM-COP) through to common workplace systems (email, web browser).

\subsection{Monitoring and Collaboration}

The main operational activities undertaken are actively monitoring for information relevant to the known priorities, identifying developments and listening for new incidents. As information is identified it is verified and corroborated using other sources.
Unfortunately there is no straight forward methodological process to verify and corroborate content. While it relies on experience and judgement, there are some guidelines that can be followed. For example, check the account details: is the account verified, when was the account created (be wary of recently created accounts); how many followers do they have; how many accounts are they following; how many tweets have they made; what are their recent tweets about; check for false reports (Google, Yahoo or snopes.com searches); check the account's Klout score. Can the account holder be found on other social media platforms, for example using Pipl People Search or Spokeo? Repeat these checks on these other platforms.

When analysing information found, check if geolocation clues are available: is the message geotagged; do their other message posts indicate their location; are significant and locatable land marks present in attached photos (Google Street View can be used) or referenced in the text. In some circumstances it may be feasible to contact the person to provide confirmation and possibly ask for further details.

Images associated with messages can be checked for authenticity using reverse image tools such as TinEye or Google Images. Image metadata can be checked (Fotoforensics) and tools can be used to see if the images have been edited (Izitru). If multiple images can be found from different sources, are they consistent? Do the known weather conditions match those observed in the photo?

Similar investigation can be performed for videos. The video can be searched on YouTube using keywords associated with the image being checked. The video thumbnail can be searched using the reverse image tools noted above. The same location checks can be done for videos as for photos: are there geocodes associated with the video; are there location clues in the associated text; and are there significant landmarks shown in the video?

A secondary process undertaken during these activities is to revise and update the list of hashtags and search terms used to find information on the social media sites. It is worth noting that different platforms will require different search terms to get the information required. This will be discovered through experience of the platforms and tools used.

\subsection{Handover}

A number of steps need to be performed as part of the end of shift. This needs to be done in a coordinated manner to ensure the smooth transition for the next person. The most important aspect of this process is one of communication; informing relevant colleagues 
that a change-over is occurring and completing all requisite note taking.

Some of the IT monitoring systems are managed with individual credentials, so they need to be exited appropriately. Unfortunately, for some systems, this means specific configuration details, notably search settings, are lost between logins. This makes the documentation of search activity crucial.

It is preferred that the hand-over from one shift of the Social Media Intelligence Analyst to another is completed in person. However this is not always possible which is why concise note taking is important.

\section{Case Studies}

The following case studies highlight the use of social media by the SCC. There were a number of other case studies that could have been described, such as a rare tornado near the Melbourne airport, an industrial crane collapse in the central business district of Melbourne and flooding events. The examples below were chosen to highlight some of the different aspects unique to intelligence gathering from social media.

\subsection{Broadmeadows Tyre Fire}

In the morning of Monday $11^{\text {th }}$ January 2016, a large fire took hold of a stock pile of tyres in the outer suburbs of Melbourne. A black smoke plume could be clearly seen causing concern for residents. This event was being reported and discussed on Twitter as shown by an example tweet in Figure 1. This user, @james howe, is asking a local radio station, @3AW693, via Twitter if they know what is causing the smoke in the photo and a short conversation followed.

At its largest extent, the fire measured approximately 100 by 30 metres and was attended to by over 100 fire fighters from the Metropolitan Fire Brigade (MFB) [20]. Water bombers helped control the blaze which was brought under control later that day, partly as a result of moving the tyres away from the fire source using bulldozers and excavators. The fire was estimated to have consumed $70 \%$ of the stockpile, about 150,000 tyres.

This event was brought to the attention of the authorities via both traditional means, calls to Triple Zero (000) the Australian Emergency Call Service, and social media. The fire brigade informed the local community to shut windows and doors and to stay indoors due to the smoke plume. There was significant particle matter in the air and the authorities were concerned that vulnerable people in the community could suffer from respiratory problems [21].

James Howe @james_howe - Jan 11

Hey @3AW693 what's on fire?

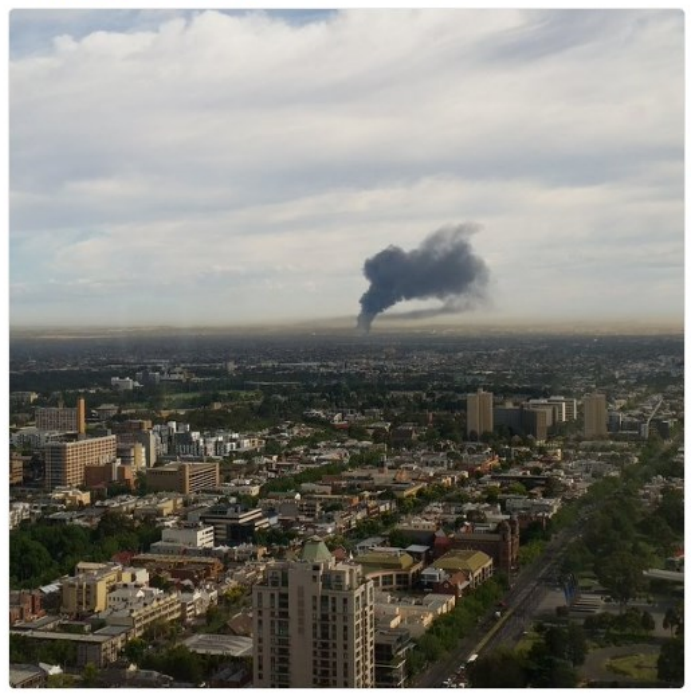

Figure 1: Early report of the tyre Fire Jan 11, 9:10 AM https://twitter.com/james howe/statuses/686309054029762561

Within the first 10 minutes of this fire the SCC Social Media Intelligence Analyst was able to find photos of the smoke plume, similar to the photo in Figure 1, followed by a closer view of the tyre dump burning in Figure 2. These images were provided to the State Response Controller, MFB Agency Commanders and the SCC Public Information Section for situational awareness.

\section{Picture from the @9NewsMelb chopper of tyre storage fire in Broadmeadows}

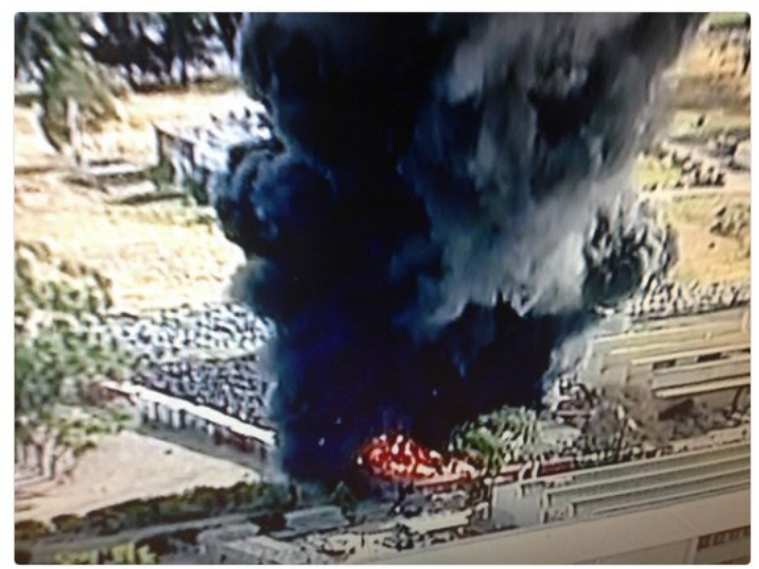

Figure 2: Local news helicopter image of the fire. https://twitter.com/andrew lund/statuses/686309420364468225 
These photos provided evidence of the source of the smoke, the potential scale of the fire that could eventuate due to the large stock pile of tyres nearby and confirmation of the prevailing weather conditions due to the direction the smoke was blowing.

This is an important point to note: while this information was used to inform and reassure the community it also provided information to the response agencies so that they could manage the event appropriately by sending the correct equipment to control the fire. This is vital to ensure the safety of the emergency services response personnel.

It is interesting to note the behaviour of people on social media for this event. In Figure 1, the user is asking a local radio station for news about a possible fire - not the emergency services. Also, Figure 2 is from a local reporter, which highlights the importance of monitoring specific high value, or key influencer, social media accounts.

\subsection{Moyston Bushfire}

In early January 2015, a bushfire in rural western Victoria burnt through over $4600 \mathrm{Ha}$ of land. Early reports of house fires were made by firefighting air and ground crews as well as media reports from helicopter news crews. The media reported their coverage extensively on social media which was monitored by the SCC. Over 90 properties were impacted with two houses destroyed [22].

There were initial reports of a tyre fire and there were concerns that it was the tyre dump near the town of Stawell that was ablaze. If this was the case, it would have serious consequences for nearby residents since it was a large dump close to town. Photos from social media, since deleted, clearly showed that the fire was in a rural setting.

The journalist who posted the tweet with an attached photo showing the fire in a grass paddock was contacted directly via social media and asked to confirm the location of the fire. She responded indicating where she thought the fire was. This information was provided to fire crews who were able to verify the location, ruling it out as being at the Stawell tyre dump. It turned out to be an illegal tyre dump which was later investigated by the Environment Protection Authority [23].

This was an example of information being obtained from a trusted social media source, a news journalist, and how the information can be verified by contacting the source. It also highlights that any information obtained from social media needs to be copied and saved locally as it can be deleted from the social media platform by the original poster after the event, as it was in this case.

\subsection{Benalla Blue-Green Algal bloom}

Australian waterways are increasingly prone to blue-green algal bloom outbreaks resulting from still waters due to drought conditions or extended dry periods combined with excess nutrients from fertilizer runoff. These blooms can cause illness in people who drink the water or eat animals from contaminated waterways, such as fish or shellfish. In some instances, theses blooms stretch for hundreds of kilometres and can be seen from space [24].

In late February 2016, an algal bloom outbreak occurred near the rural Victorian town of Benalla. The public were warned of the danger and advised to not make direct contact with the water. Warning signs were posted at the major recreational areas and Twitter and Facebook were also used to inform the community, including URL references to further information available on relevant websites and VicEmergency [25].

The SCC Social Media Intelligence Analyst reviewed community reactions during this time, monitoring the questions and answers on the official social media account and checking other community self-help blogs or forums discussing this incident. They were also interested in the uptake as a news item in the local media.

There were a number of findings discovered broadly categorized as:

- Requests for more information.

People wanted to know what an algal bloom was, what caused it, how long it would last, if it would travel down the waterways, how it affects irrigation and so on.

- Impact.

How will it affect them directly - can they still go camping or water-skiing?

- Concern.

People expressing their concern of drinking the water or of pets getting sick or dying.

- Scepticism.

Disregard of warnings, saying it is a low risk and complaining about a 'nanny state'.

Note that the social media intelligence analyst was not involved in communicating with the public via social media about these issues. Rather, these conversations were monitored along with other information sources to understand how the public were responding to this public health incident. As a result, there were changes to the public messaging.

\section{Related Work}

The utility of social media as an information source for first responders has been recognized for situational 
awareness and decision making. The report from the Virtual Social Media Working Group and DHS First Responders Group [1] provides examples of how agencies currently leverage social media, its potential applications and the challenges likely to be encountered. They identify research areas to address technological, organizational and policy issues. In relation to social media content, they specifically highlight the need to access, share and integrate information, privacy and legal considerations and operational requirements such as the importance of geo-located and temporal characteristics of content.

An overview of the main uses of Twitter in emergency management over the past ten years found several uses across the disaster lifecycle. This includes the identification of hazards, community engagement to prepare for disasters, early warning communication, real-time crowdsourcing, emotional support, identifying needs in communities and resource allocation during the recovery phase [12]. They also identified some currently underutilized uses of Twitter such as social capital formation, capacity building, virtual communities of best practice and social change.

It is recognised that there are currently operational and organisational issues in the use of social media within emergency service agencies. A semi-structured survey interview was conducted to identify these issues by U.S. public sector emergency managers [2]. They found there is limited personal time to use social media, a lack of organisational policies and guidelines for its use and concern over the trustworthiness of data. For these reasons many organisations prohibit the use of social media for intelligence gathering purposes [2].

Similarly, while social media has been recognised as a new data channel to receive public crowdsourced information about emergency events other research confirms that its adoption is not yet widespread [13]. Again, this is due to a number of limitations: how to identify relevant information from the large amount of available content, ensuring the veracity of what is found and reliably inferring the location of the event to coordinate an appropriate response. These issues need to be mindful of the needs of crisis coordinators and emergency managers to make operational decisions based on the best available information.

Other research [3] focused on the needs of emergency management during disaster events. They found that there is no single tool that currently meets all the needs of incident controllers and they identified a number of areas for tooling improvements, such as the automated evaluation of information sources, the ability to follow social media content from specific individuals, the geographic representation of search results, access to real-time feeds across multiple platforms, and the flexibility to modify searches.
The role and use of social media and bystander reports for making sense of an emergency event has been investigated using a framing analysis [26]. They found useful insights into how social media is used and perceived along with how bystander reports are interpreted which impacts on practice.

Other case studies gain insight by conducting interviews with emergency management personnel after crisis situations [9,14]. We believe our work is unique in that is draws upon operational experience from a practitioner who has been responsible for instituting a new role of the social media intelligence analyst in a crisis coordination centre who has also delivered training sessions to impart skills in the effective use of social media for intelligence gathering.

\section{Conclusions}

The Social Media Intelligence Analyst is a new operational role within a State Control Centre in Australia dedicated to obtaining situational awareness from social media to support decision making for emergency management. We have outlined where this role fits within the structure of a command and control organisation for emergency management and importantly, we have provided a comprehensive overview of the operational tasks undertaken in performing this role. We have also provided three example uses cases which highlight the utility of this role in an emergency management context.

This is a relatively new role within the SCC that will be monitored, evaluated and refined over time as a result of reviews after operational activity. It is hoped that other jurisdictions adopt similar practices and that in time this role will be formally recognised within the standard operating models currently used in the Australian emergency management community.

\section{References}

[1] Using Social Media for Enhanced Situational Awareness and Decision Support. Virtual Social Media Working Group and DHS First Responders Group. June 2014.

http://goo.gl/WiSd3C [Accessed: 27 April 2016].

[2] S.R. Hiltz, J. Kushma and L. Plotnick. "Use of Social Media by US Public Sector Emergency Managers: Barriers and Wish Lists". In $11^{\text {th }}$ International Conference on Information Systems for Crisis Response and Management (ISCRAM), Pennsylvania, USA. May 2014.

[3] C. Mason and R. Power. "Improving social media monitoring and analysis tools for emergency management". In Weber, T., McPhee, M.J. and Anderssen, R.S. (eds) MODSIM2015, $21^{\text {st }}$ International Congress on Modelling 
and Simulation. Modelling and Simulation Society of Australia and New Zealand, December 2015, pp. 1195-1201.

[4] L. Plotnick, S.R. Hiltz, J.A. Kushma, A. Tapia "Red Tape: Attitudes and Issues Related to Use of Social Media by U.S. County-Level Emergency Managers". In $12^{\text {th }}$ International Conference on Information Systems for Crisis Response and Management (ISCRAM), Kristiansand, Norway, May 2015.

[5] D. Havlik, J. Pielorz, A. Widera "Interaction with citizens experiments: from context-aware alerting to crowdtasking". In $13^{\text {th }}$ International Conference on Information Systems for Crisis Response and Management (ISCRAM) Rio de Janeiro, Brasil, May 2016..

[6] S Vieweg, AL Hughes, K Starbird, L Palen. "Microblogging during two natural hazards events: what twitter may contribute to situational awareness" Proceedings of the SIGCHI conference on human factors in computing systems. Atlanta, Georgia, USA: ACM. pp 1079-1088. 2010.

[7] A. Bruns and J. Burgess "Crisis Communication in Natural Disasters: The Queensland Floods and Christchurch Earthquakes". In K. Weller, A. Bruns, J. Burgess, M. Mahrt \& C. Puschmann (eds.) Twitter and Society, Peter Lang Publishing, New York, 2014, pp 373-384.

[8] AL Hughes, L Palen. "Twitter adoption and use in mass convergence and emergency events" International Journal of Emergency Management 6 (3-4), 248-260. 2009.

[9] Fabio Ciravegna, Suvodeep Mazumdar, Neil Ireson, \& Peter Cudd. "Seeing through the Eyes of the Citizens during Emergencies". In $13^{\text {th }}$ International Conference on Information Systems for Crisis Response and Management (ISCRAM) Rio de Janeiro, Brasil, May 2016.

[10] F. Vis, S. Faulkner, K. Parry, Y. Manyukhina and L. Evans "Twitpic-ing the riots: analysing images shared on Twitter during the 2011 UK riots”. In: Weller, K., Bruns, A., Burgess, J., Mahrt, M. and Puschmann, C., (eds.) Twitter and Society. Peter Lang Publishing, New York, 2014 pp 385-398.

[11] S. Daly and J. Thom "Mining and Classifying Image Posts on Social Media to Analyse Fires" In $13^{\text {th }}$ International Conference on Information Systems for Crisis Response and Management (ISCRAM), Rio de Janeiro, Brazil, May 2016.

[12] Twitter turns ten: its use to date in disaster management, Neil Duffy. Australian Journal of Emergency Management, vol. 31, no. 2, pp. 50-54, April 2016.

https://ajem.infoservices.com.au/items/AJEM-31-02-10

[13] B. Lindsay "Social Media and Disasters: Current Uses, Future Options, and Policy Considerations" September 2011
[14] Emma Potter. "Balancing conflicting operational and communications priorities: social media use in an emergency management organization". In $13^{\text {th }}$ International Conference on Information Systems for Crisis Response and Management (ISCRAM) Rio de Janeiro, Brasil, May 2016.

[15] Shane Errol Halse, Andrea Tapia, Anna Squicciarini, \& Cornelia Caragea. "Tweet Factors Influencing Trust and Usefulness During Both Man-Made and Natural Disasters" In $13^{\text {th }}$ International Conference on Information Systems for Crisis Response and Management (ISCRAM) Rio de Janeiro, Brasil, May 2016.

[16] Catherine Stephenson, John Handmer, and Aimee Haywood. Estimating the net cost of the 2009 Black Saturday fires to the affected regions. Technical Report, RMIT, Bushfire CRC, Victorian DSE, Feb 2012.

[17] R. Oloruntoba. "Plans never go according to plan: An empirical analysis of challenges to plans during the 2009 Victoria bushfires" Technological Forecasting and Social Change,80,9,1674-1702, 2013, North-Holland

[18] Australian Emergency Management Manual series. Australian Emergency Management Institute. 2004.

[19] The Australian Inter-Service Incident Management System, Fourth Edition (AIIMS-4). 2013.

[20] Melbourne industrial fire. Jan 2016. At http://goo.gl/eV7DEl [Accessed: 13 May 2016].

[21] Broadmeadows tyre fire controlled, smoke a concern. Jan 2016. http://goo.gl/rzG1fd [Accessed: 13 May 2016].

[22] Moyston still feeling fire impact says MP Emma Kealy. Feb 2015. http://goo.gl/csJjEd [Accessed: 15 June 2016].

[23] Moyston fire: Dumping investigated after 30,000 tyres catch fire. Jan 2015. http://goo.gl/rDI0Wl [Accessed: 15 June 2016]

[24] Watch out, Australia: a red-hot summer means bluegreen algae. October 2015. http://goo.gl/ZGlm6C

[Accessed 15 June 2016].

[25] VicEmergency. http://emergency.vic.gov.au/respond/

[26] F. Bergstrand and D. Stenmark "Leveraging Bystander Reports in Emergency Response Work: Framing Emergency Managers Social Media Use" $49^{\text {th }}$ Hawaii International Conference on System Sciences (HICSS), Koloa, HI, 2016, pp. 162-171. 